\title{
Technology and opportunities of photon sieve CubeSat with deployable optical membrane
}

\author{
Hyun Jung Kim¹, Shravan Hariharan², Matthew Julian³, and David Macdonnell ${ }^{4}$ \\ 1. National Institute of Aerospace (NIA), 100 Exploration Way, Hampton VA 23666 \\ 2. Aerospace Engineering, Georgia Institute of Technology, Atlanta, GA 30332 \\ 3. University of Virginia, Charlottesville, VA 22904 \\ 4. NASA Langley Research Center, Hampton VA 23681
}

\begin{abstract}
A photon sieve is a revolutionary optical instrument that provides high resolution imaging at a fraction of the weight of typical telescopes, with an areal density of $0.3 \mathrm{~kg} / \mathrm{m}^{2}$ compared to $25 \mathrm{~kg} / \mathrm{m}^{2}$ for the James Webb Space Telescope. The photon sieve is a variation of a Fresnel Zone Plate consisting of many small holes arranged in a series of concentric rings. The sieve works by diffracting light of a certain wavelength to a specified focal point for imaging, so that only a specific wavelength can be imaged. Moreover, the better image contrast and higher signal-to-noise ratios come from suppressing higher diffracted orders by apodizing the number of pinholes in the outer rings. Finally, a photon sieve requires less supports and can withstand more deformation without a reduction in the imaging qualities.

Due to these properties, various groups have created PS CubeSats for Earth and Sun imaging at a low cost and weight specifically using deployable technology. A team at the Air Force Research Laboratory created a design and prototype of a mechanism that deploys a $20 \mathrm{~cm}$ diameter photon sieve. The United States Air Force Laboratory used a similar design to create a CubeSat-based deployable photon sieve. The team at NASA Langley Research Center has researched photon sieves for conducting an Earth-observing experiment using LIDAR (Light Detection and Ranging) with a higher signal-to-noise ratio benefit from the PS. This paper provides a state of the art overview on existing PS CubeSat technology with deployable structures and applications. Especially, the paper introduces PS for LIDAR applications and discusses CubeSat-based PS challenge being worked at the NASA Langley Research Center.
\end{abstract}

Keywords: CubeSat, photon sieve (PS), deployable optical membrane, imaging, telescope for LIDAR 


\section{About the Photon Sieve (PS)}

Photon sieves (PS), proposed in 2001 by Kipp et. al., are a planar diffractive optical element based on the well known Fresnel zone plate, in which the Fresnel zone plate (FZP) rings are essentially broken up into individual pinholes as shown in Figure 1 [1]. The contribution of each pinhole to the overall focal point intensity follows a first-order Bessel function, and is given by the equation (1) [2].

$$
F \propto \frac{d}{w} J_{1}\left(\frac{\pi d}{2 w}\right)
$$

where $F$ is the overall focal point intensity contribution of a single pinhole, and $d$ and $w$ are the pinhole diameter and underlying Fresnel zone width, respectively. As can be seen from equation (1), the radio $d / w$ can take an infinite number of values $(1.53,3.53,5.53, \ldots)$ such that the Bessel function is maximized. Therefore, the diameter of the photon sieve pinholes can be made larger than the underlying Fresnel zone width.
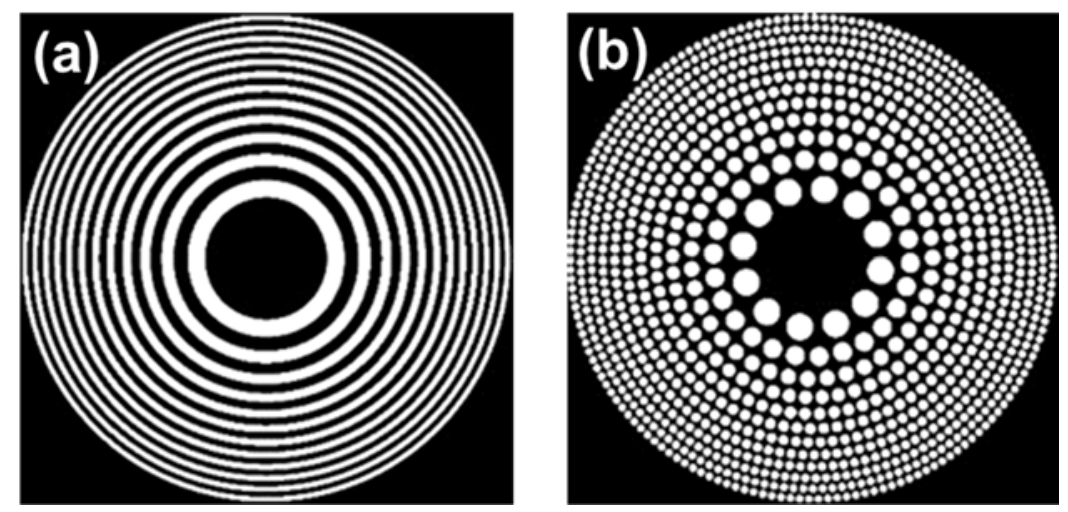

Figure 1. Designs of (a) Fresnel zone plate and (b) photon sieve with same 12 ring numbers

Similarly to FZPs, PS's converge incident light to a designed focal point via optical diffraction. However, PSs have the added benefit of suppressing higher diffracted orders by apodizing the number of pinholes in the outer rings, which leads to better image contrast and higher signal-to-noise ratios [3-7]. The flexibility in pinhole diameter also relaxes fabrication constraints, meaning that a given fabrication system will be able to produce a higher numerical aperture (NA) photon sieve than zone plate. Thus, a photon sieve requires less supports and can withstand more deformation without a reduction in the imaging qualities. In addition, because the maximum resolution of photon sieves and Fresnel zone plates is defined by the width of the outermost zone, photon sieves are able to achieve a higher resolution than the corresponding Fresnel zone plate. The main drawback in using photon sieves is their inherently lower diffraction efficiency compared to zone plates. However, photon sieve phase structures have recently been investigated and have reached efficiencies of $10 \%$ [8]. With the use of multilevel phase structures, photon sieve efficiencies are 
greatly improved up to $25 \%$ [9]. Due to these properties and technical improvement, various groups have created PS CubeSats for Earth and Sun imaging at a low cost and weight specifically using deployable technology.

\section{CubeSat Project}

Large launch vehicles often contain additional volume or mass not occupied by the primary payload, and it soon became feasible to add small satellites to these launches as secondary payloads. However, the satellites varied drastically in size and mass, and it became necessary to devise a standard for small satellites including picosatellite development. The CubeSat Project started in 1999 as a joint venture between Stanford University and California Polytechnic State University (Cal Poly), and is a collaborative project that provides a design standard for picosatellite development [10]. These picosatellites, known as CubeSats, are considerably cheaper than larger satellites, and often carry scientific payloads. CubeSat size is measured in terms of units (U), and the basic unit, $1 \mathrm{U}$, measures $10 \mathrm{~cm}$ x $10 \mathrm{~cm}$ x $10 \mathrm{~cm}$. CubeSats are scaled in multiples of the $1 \mathrm{U}$ form factor; to date, $1 \mathrm{U}, 1.5 \mathrm{U}(15 \mathrm{~cm} \times 10 \mathrm{~cm} \times 10 \mathrm{~cm}), 2 \mathrm{U}(20 \mathrm{~cm} \times 10 \mathrm{~cm} \times 10 \mathrm{~cm})$, and $3 \mathrm{U}(30 \mathrm{~cm} \times 10 \mathrm{~cm} \times 10 \mathrm{~cm})$ CubeSats have launched. Larger CubeSats, such as a $6 \mathrm{U}(30 \mathrm{~cm} \times 20 \mathrm{~cm}$ $\mathrm{x} 10 \mathrm{~cm}$ ) have been proposed and designed, but have not launched to date. A $1 \mathrm{U}$ CubeSat has a maximum mass of $1.33 \mathrm{~kg}$, a $1.5 \mathrm{U}$ CubeSat has a maximum mass of $2.00 \mathrm{~kg}$, the maximum mass of a $2 \mathrm{U}$ CubeSat is $2.66 \mathrm{~kg}$, and the maximum mass of a $3 \mathrm{U}$ CubeSat is $4.00 \mathrm{~kg}$. The maximum mass of a $6 \mathrm{U}$ CubeSat is 12.00 $\mathrm{kg}$ [11]. Due to the limited volume available to CubeSats, a majority are constructed as $1 \mathrm{U}$, $2 \mathrm{U}$, or $3 \mathrm{U}$ satellites. However, certain $6 \mathrm{U}$ satellites have been selected to launch in the near future. Table 1 summarizes the selected $6 \mathrm{U}$ missions that have already been manifested to launch.

\begin{tabular}{|c|c|c|c|c|}
\hline Mission Name & $\begin{array}{c}\text { Year } \\
\text { Selected }\end{array}$ & University/Group & Mission Objective & $\begin{array}{c}\text { Launch Vehicle } \\
\text { (Launch date) }\end{array}$ \\
\hline LAICE & 2012 & $\begin{array}{l}\text { University of Illinois, } \\
\text { Urbana-Champaign }\end{array}$ & $\begin{array}{c}\text { To perform } \\
\text { atmospheric gravity } \\
\text { wave studies via in- } \\
\text { situ measurements } \\
\text { of wave } \\
\text { perturbations in the } \\
\text { ionosphere and }\end{array}$ & $\begin{array}{c}\text { ELaNa-22 (August } \\
1,2017)\end{array}$ \\
\hline
\end{tabular}




\begin{tabular}{|c|c|c|c|c|}
\hline & & & $\begin{array}{l}\text { remote sensing of } \\
\text { the middle } \\
\text { atmosphere [12] }\end{array}$ & \\
\hline RBLE & 2012 & $\begin{array}{c}\text { NASA Goddard } \\
\text { Space Flight Center }\end{array}$ & $\begin{array}{c}\text { Consists of a } \\
\text { compact relativistic } \\
\text { electron proton } \\
\text { telescope and a } \\
\text { visible light } \\
\text { photometer to } \\
\text { measure radiation } \\
\text { belts [13] }\end{array}$ & $\begin{array}{c}\text { ELaNa-22 (August } \\
1,2017)\end{array}$ \\
\hline EcAMSat & 2013 & $\begin{array}{c}\text { NASA Ames } \\
\text { Research Center }\end{array}$ & $\begin{array}{c}\text { EcAMSat will } \\
\text { investigate } \\
\text { spaceflight effects } \\
\text { on bacterial } \\
\text { antibiotic resistance } \\
\text { and its genetic basic } \\
{[14]}\end{array}$ & $\begin{array}{c}\text { ELaNa-XIII (Date } \\
\text { TBD) }\end{array}$ \\
\hline RASCAL & 2013 & St. Louis University & $\begin{array}{c}\text { Demonstrates key } \\
\text { technologies for } \\
\text { proximity } \\
\text { operations and } \\
\text { space situational } \\
\text { awareness: infrared } \\
\text { imaging, 6DOF } \\
\text { propulsion, RF } \\
\text { proximity sensing, } \\
\text { and automated } \\
\text { operations [15] }\end{array}$ & $\begin{array}{c}\text { ELaNa-23 } \\
\text { (April 2018) }\end{array}$ \\
\hline
\end{tabular}




\begin{tabular}{|c|c|c|c|c|}
\hline ANDESITE & 2014 & Boston University & $\begin{array}{l}\text { This small network } \\
\text { of miniature } \\
\text { CubeSats will } \\
\text { attempt to resolve } \\
\text { current densities at } \\
\text { varying spatial } \\
\text { resolutions in the } \\
\text { near-Earth } \\
\text { magnetosphere } \\
\text { using } \\
\text { measurements from } \\
\text { Anisotropic } \\
\text { Resistance } \\
\text { Magnetometers } \\
\text { [16] }\end{array}$ & $\begin{array}{c}\text { ELaNa-XIX } \\
\text { (November 2017) }\end{array}$ \\
\hline TEMPEST-D, 1 & 2015 & $\begin{array}{c}\text { Colorado State } \\
\text { University }\end{array}$ & $\begin{array}{l}\text { Demonstrates a } \\
\text { millimeter-wave } \\
\text { radiometer for } \\
\text { NASA's Earth } \\
\text { Science goals [17] }\end{array}$ & $\begin{array}{c}\text { ELaNa-23 (April } \\
\text { 2018) }\end{array}$ \\
\hline RainCube & 2016 & $\begin{array}{l}\text { Jet Propulsion } \\
\text { Laboratory }\end{array}$ & $\begin{array}{c}\text { Technology } \\
\text { demonstration } \\
\text { mission to enable } \\
\text { Ka-band } \\
\text { precipitation radar } \\
\text { and deployable } \\
\text { antenna on CubeSat } \\
\text { [18] }\end{array}$ & $\begin{array}{c}\text { ELaNa-23 (April } \\
\text { 2018) }\end{array}$ \\
\hline
\end{tabular}




\begin{tabular}{|c|c|c|c|c|}
\hline SORTIE & 2016 & $\begin{array}{c}\text { Atmospheric \& } \\
\text { Space Technology } \\
\text { Research Associates }\end{array}$ & $\begin{array}{l}\text { Objective is to } \\
\text { discover the } \\
\text { sources of wave- } \\
\text { like plasma } \\
\text { perturbations in the } \\
\text { F-region } \\
\text { ionosphere, and to } \\
\text { determine the } \\
\text { method of the } \\
\text { perturbation } \\
\text { formation [19] }\end{array}$ & $\begin{array}{c}\text { ELaNa-23 (April } \\
\text { 2018) }\end{array}$ \\
\hline CubeRRT & 2016 & $\begin{array}{c}\text { The Ohio State } \\
\text { University }\end{array}$ & $\begin{array}{l}\text { To demonstrate } \\
\text { wideband radio } \\
\text { frequency interface } \\
\text { for spaceborne } \\
\text { microwave } \\
\text { radiometers [20] }\end{array}$ & $\begin{array}{c}\text { ELaNa-23 (April } \\
\text { 2018) }\end{array}$ \\
\hline
\end{tabular}

Table 1: Manifested 6U CubeSat Launches

In addition to the launches mentioned in Table 1, seven other $6 \mathrm{U}$ CubeSat missions are in development but do not have launch dates. One of the missions consists of two $6 \mathrm{U}$ CubeSats, bringing the total number to eight $6 \mathrm{U}$ CubeSats under development and awaiting launch opportunities [21].

The stringent size and mass requirements are to ensure that each CubeSat fits within the launch vehicle without influencing the mission. Due to the miniaturization, CubeSats enable many missions that large satellites performed in the past [21] and mission applications previously achievable only by large satellites became target applications for CubeSats. While the satellite has decreased in size, some satellite components that require collecting or reflecting science signals [22], photons, electromagnetic (EM) radiation, and numerous other large aperture devices must necessarily remain large, for example, telescope lenses, communication antennas, solar sails, drag sails, instrument booms, and photovoltaic power arrays [23]. Eventually, CubeSats need miniaturized deployment systems and materials to design more functional CubeSats. In addition to the mission payload, CubeSats contain various subsystems that control their flight 
and operation. These subsystems include Command and Data Handling (C\&DH), Electrical Power Systems (EPS), Communications, Attitude Determination and Control (ADCS), Propulsion, Structure, and Thermal Control. Each subsystem, as well as the state of the art technology, is described in the NASA Small Spacecraft Technology State of the Art report [24,25].

\section{State of the art PS CubeSat}

Various groups have created photon sieve (PS) CubeSats for Earth and Sun imaging or science signal detecting by using deployable technology and Lidar concept. A team at the Air Force Research Laboratory (AFRL) created a design and prototype of a mechanism that deploys a $20 \mathrm{~cm}$ diameter photon sieve and the United States Air Force Laboratory (USAF) used a similar design to create a CubeSat-based deployable photon sieve. Other group at NASA Ames Research Center (NASA ARC) designed a 6U CubeSat for a deployable telescope; this design is not for a photon sieve, but can be applied to PS CubeSat missions. Finally, NASA Langley Research Center (NASA LaRC) creates PS's for science signal detection from the atmosphere. Each design will be described in detail in the following sections as well as the challenges facing each approach to make the mission a success.

\subsection{PS CubeSat at Air Force Research Laboratory}

The Air Force Research Laboratory (AFRL) Space Vehicle Directorate, based in New Mexico, collaborated with LoadPath, a local company, to design a photon sieve deployment system [26]. This study dealt with the mechanical system for deployment, and requirements necessary to meet a successful deployment. The "sieve" used in testing was simply a $20 \mathrm{~cm}$ diameter Kapton membrane, without the additional holes that a real photon sieve would have and the research is less focused on the optics and imaging by using the PS. The structure, shown in its stored form in Figure 2, consists of three composite tape springs, rolled around drums. The photon sieve membrane is folded and placed in a cylinder in the middle of these three drums. 


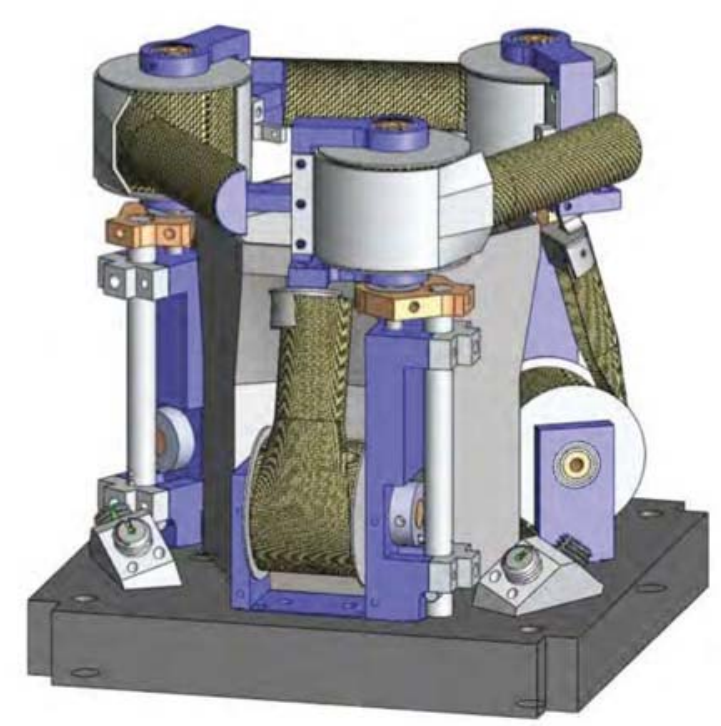

Figure 2. Drawing of tape Spring Deployment System (stored)

These tape springs, similar in form to a carpenter's measuring tape, are able to self-hinge and elastically deploy, with enough compressive strength to support the sieve. These three tape springs are coupled with three graphite lanyards for tensile strength to form a hexapod structure. The hexapod structure supports a membrane, which is a single piece consisting of the photon sieve as well as the support contact points. A hole is cut in the center of the photon sieve to prevent radial wrinkles when folding. Springs at each corner stretch the membrane to an appropriate tension that keeps the sieve flat enough for accurate measurement. This system is shown in its deployed form in Figure 3 and 4.

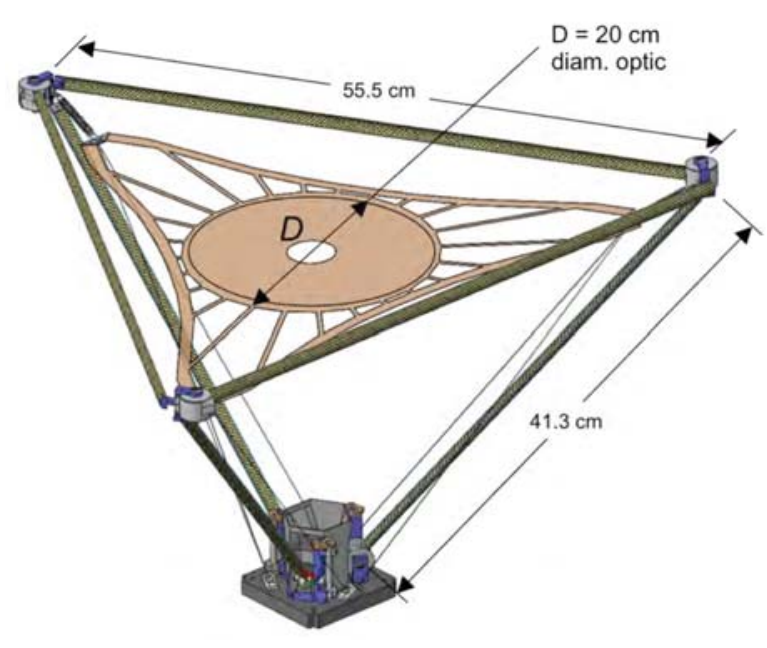

Figure 3. Drawing of tape Spring Deployment System (deployed) 

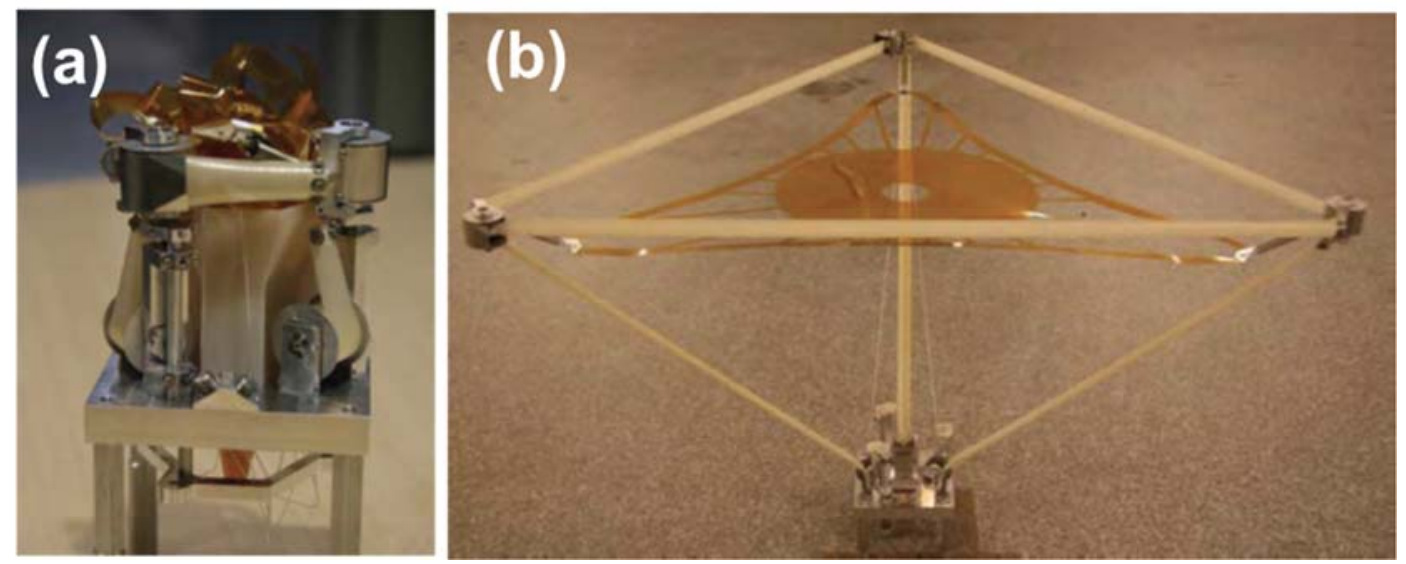

Figure 4. AFRL Photon Sieve Test (a) in stored and (b)deployed forms

The whole system fits into $1 \mathrm{U}$ and deployment is controlled by one single and vertical shaft mechanism resulting in a synchronous release. A prototype of CubeSat was constructed for deployment tests and the mechanism was worked functionally in the test facility, although microgravity testing as well as other dynamic testing is necessary in the future. As for the optics, this mission used a membrane solely, not a PS for the mechanical properties. Therefore, the optics accuracy could not be measured. However, by measuring the in-plane and out-of-plane deformations of the "sieve", the study was able to determine the feasibility of the deployment system, using calculated maximum deformations as a baseline. This system's strength lie in its small size and light weight, as those are essential for packaging in the CubeSat form factor. However, by packaging the photon sieve in a small cylinder, the risk increases of forming permanent material wrinkles in the membrane, that remain in the material even with tension at the corners. This would result in inaccurate imaging, which directly violates the primary mission goal. In addition, the system is complex to manufacture.

This experiment's goal was to prove the feasibility of the deployment system, the system was not implemented into a CubeSat yet, and the technology has not been demonstrated in a real-world application.

\subsection{FalconSAT-7: PS CubeSat at United States Air Force Academy}

A team consisting of the United States Air Force Academy as well as researchers from NASA Goddard Space Flight Center extended the previous concept to construct a CubeSat named the FalconSat7 to launch in 2018. FalconSat-7 is a $3 \mathrm{U}$ CubeSat housing Peregrine, a 1.5U deployable photon sieve telescope [3]. The mission's goal is to use H-alpha rays from sunlight to image the solar surface. H-alpha 
rays are visible light rays with a wavelength of $656.4 \mathrm{~nm}$, and are often used by astronomers due to their abundance in star emissions. The CubeSat was designed to conduct imaging, the emphasis was on the optics rather than the deployment system, which was outsourced to MMA Design LLC. The specific wavelength used in this mission was selected because even with a narrow bandwidth and low efficiency, there would still be enough photon flux to obtain images in low-light situations [4]. The prototype master sieve was a phase PS, which meant that rather than using holes, the manufacturer used raised circular pads on a transparent substrate. This PS was made out of chrome-coated quartz. The membrane photon sieve for the CubeSat is made out of aluminum-coated 28-micron Kapton polyimide, and has a diameter of $20 \mathrm{~cm}$. The researchers originally planned to use Novastrat, a polymer with a near-zero coefficient of thermal expansion, as the substrate material, but tests showed that Novastrat was too brittle to survive the deployment process. The focal length of the sieve is $40 \mathrm{~cm}$. This telescope is almost identical to the AFRL system in terms of structure, except the composite tape springs are replaced by three spring-loaded pantographs. To deploy the pantographs, a current is run, which melts a wire locking the containment door. This allows the elastically loaded pantographs to push through the opening and deploy. Figures 5 and 6 show this system in the stowed and deployed modes. The sieve itself is stored in a cylinder that is $5 \mathrm{~cm}$ in diameter and $7.8 \mathrm{~cm}$ tall.

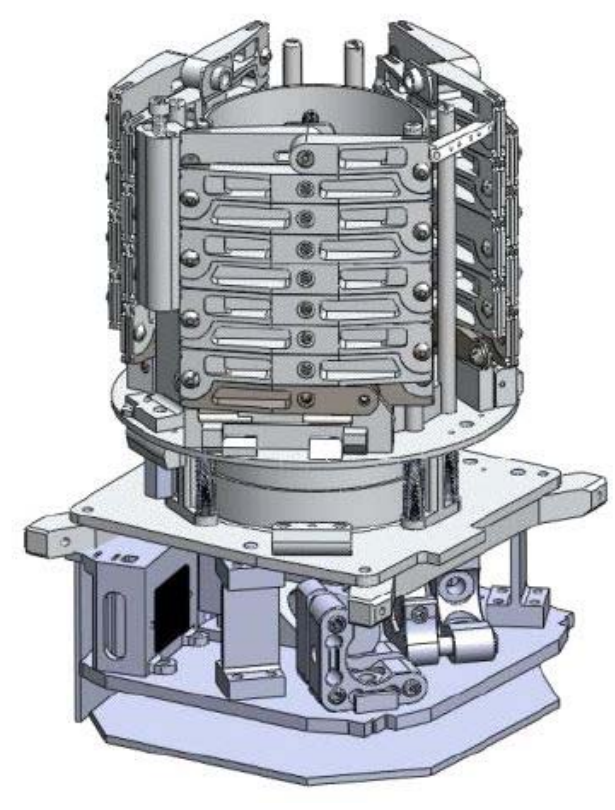

Figure 5. Drawing of peregrine deployment system (stowed) 


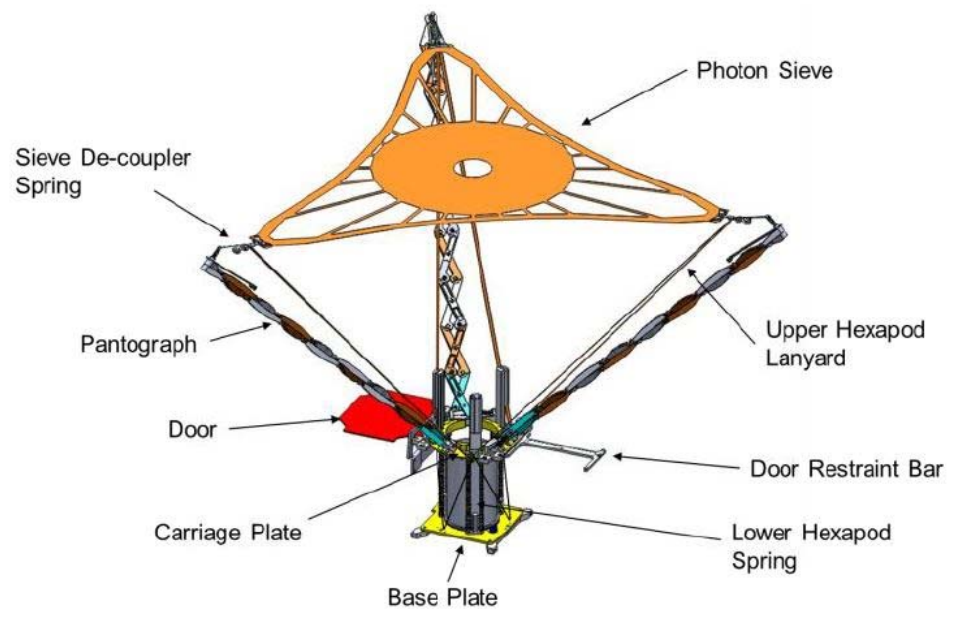

Figure 6. Peregrine Deployment System (deployed)

Underneath the sieve, the system contains secondary optics that filter the H-alpha rays, and then focus the signal on a CCD science camera as shown in Figure 7 [5]. When the light passes through the photon sieve, it moves to a lens, which collimates the beam to pass through a filter. Although the photon sieve is designed to focus the H-alpha rays, it is necessary to include a narrow band filter due to the magnitude of light radiation from the Sun. This H-alpha filter prevents unfocused light of different wavelengths from reaching the CCD camera, so that the target $\mathrm{H}$-alpha rays are the only signal being read [6]. After passing through the interference filter, the light is focused again to the CCD camera. The benefits of this system are that it fits into $1.5 \mathrm{U}$ even with secondary optics, leaving $1.5 \mathrm{U}$ for the satellite's flight systems and avionics. In addition, the team has conducted microgravity testing, which has confirmed that the deployment system works under zero-gravity conditions [7]. However, the use of pantographs adds a considerable amount of weight to the system, and the risk still remains of damaging the photon sieve optic during packaging and transport.

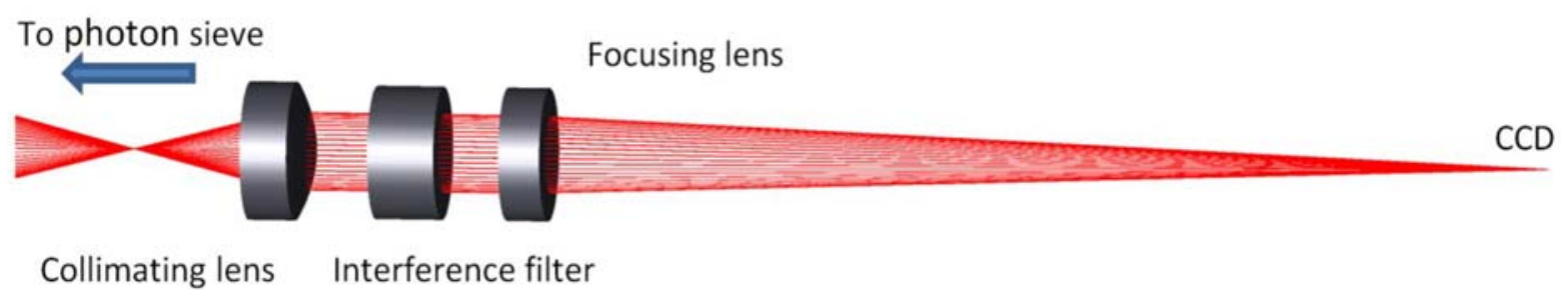




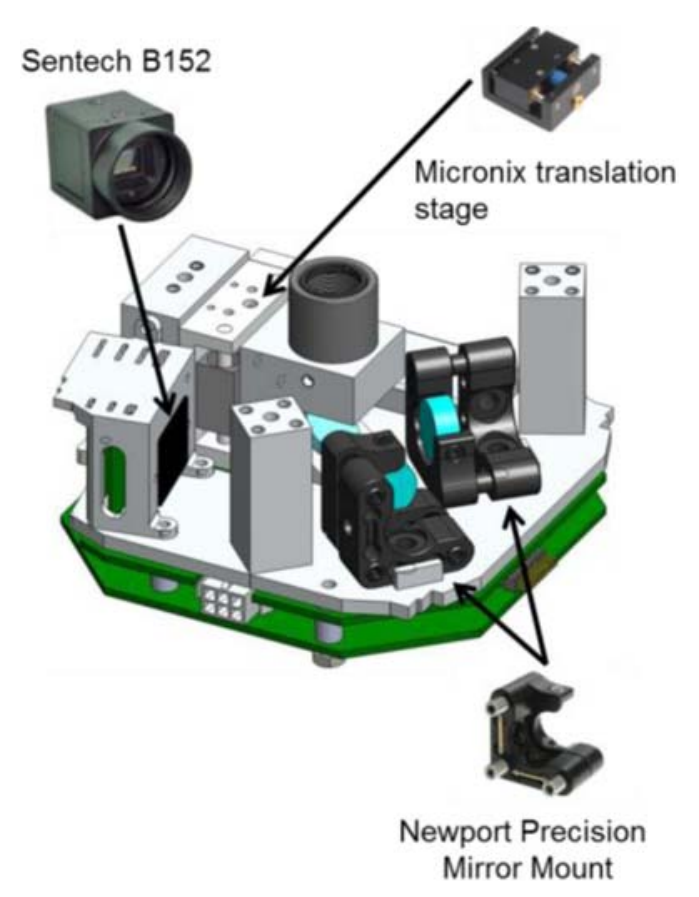

Figure 7. Peregrine Secondary Optics System schematic (top) and 3-D Design (bottom)

The team conducted imaging from Earth using a breadboard prototype along with both the glass photon sieve and secondary focusing optics. Figure 8 a photo of the sun taken on July 31, 2012 by using the Air Force Academy photon sieve and Solar Dynamics Observaroty (SDO). The image taken by photon sieve closely matches the resolution and Field of View (FOV) of SDO image taken by the same day as shown in Figure 8(a).
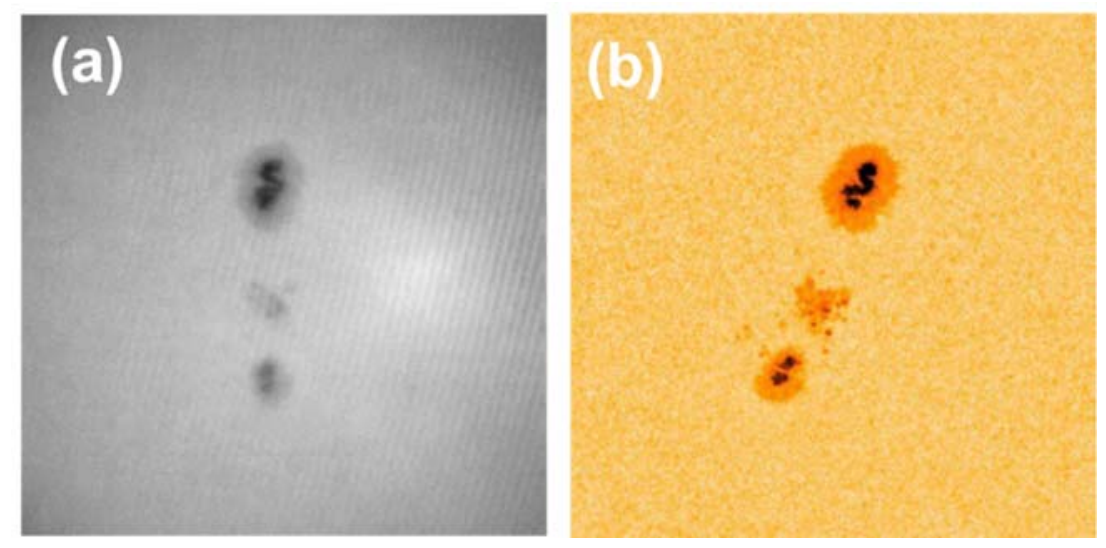

Figure 8. Solar image taken by (a) FalconSat-7 photon sieve and (b) Solar Dynamics Observatory (SDO) (image credit: USAFA) 
As seen in Figure 8, the photon sieve optical system was able to capture a high-resolution image, which confirms that this optical system is feasible for space applications. Unlike the Air Force Research Lab deployment tests, which focused on solely the deployment system, the FalconSAT-7 tests ensured that the imaging spacecraft as a whole was ready for flight.

\subsection{CubeSat concept with Deployable Truss at NASA Ames Research Center}

Unlike the other projects described, the Collapsible Telescope proposed by NASA Ames Research Center and collaborating groups was designed for a reflective telescope rather than a diffractive optic such as a photon sieve. Researchers from NASA Ames Research Center, with collaboration with the National Society for Black Engineers (NSBE), NASA Johnson Space Center, San Jose State University, and Stanford University, conducted a study to determine the feasibility of a collapsible space telescope [27]. This telescope was designed to fit into a $6 \mathrm{U}$ CubeSat, with the optics occupying $4 \mathrm{U}$ of space. This leaves $2 \mathrm{U}$ for the CubeSat avionics and hardware.

The telescope was not designing for mounting a photon sieve; the study concerned a Ritchey-Chrétien reflective telescope, which consists of two mirrors for imaging. However, due to the rigid shape with 15$20 \mathrm{~cm}$ diameter size of the optic, this technology could be used for a photon sieve application.

The study considered three different technologies for the telescope: a strain-deployed truss, an inflatable structure, and a scissor-lift structure, similar to the FalconSAT-7 Pantograph. Table 2 shows the decision matrix of the trade study, which weighs the pros and cons of each technology.

(Scale - based on $+1,0$ and $-1 ;+1$ is best)

\begin{tabular}{|l|l|c|c|c|}
\hline FOM & Weight & Coilable & Inflatable & Scissor Lift \\
\hline $\begin{array}{l}\text { Structural } \\
\text { Rigidity }\end{array}$ & 0.125 & 1 & 0.5 & 0 \\
\hline Reliability & 0.125 & 1 & -0.5 & 0 \\
\hline Packing Ratio & 0.200 & 0 & 1 & -1 \\
\hline Energy Efficiency & 0.125 & 1 & 0.5 & 0.5 \\
\hline $\begin{array}{l}\text { Simplicity to } \\
\text { Manufacture }\end{array}$ & 0.100 & 0 & 1 & 0.5 \\
\hline Cost & 0.100 & 1 & 0 & 1 \\
\hline Performance & 0.125 & 1 & 0.5 & 1 \\
\hline Mass & 0.100 & 1 & 0 & .5 \\
\hline Total & $\mathbf{1 . 0 0 0}$ & $\mathbf{0 . 7}$ & $\mathbf{0 . 4 2 5}$ & $\mathbf{0 . 1 8 7 5}$ \\
\hline
\end{tabular}

Table 2: Decision matrix for deployable boom concepts 
The strain-deployed truss, labeled as Coilable in the Table 2, was determined to be the best solution by far. The strain-deployed truss skeleton consists of three main components, Batten rings, longerons, and diagonal stringers as shown in Figure 9.

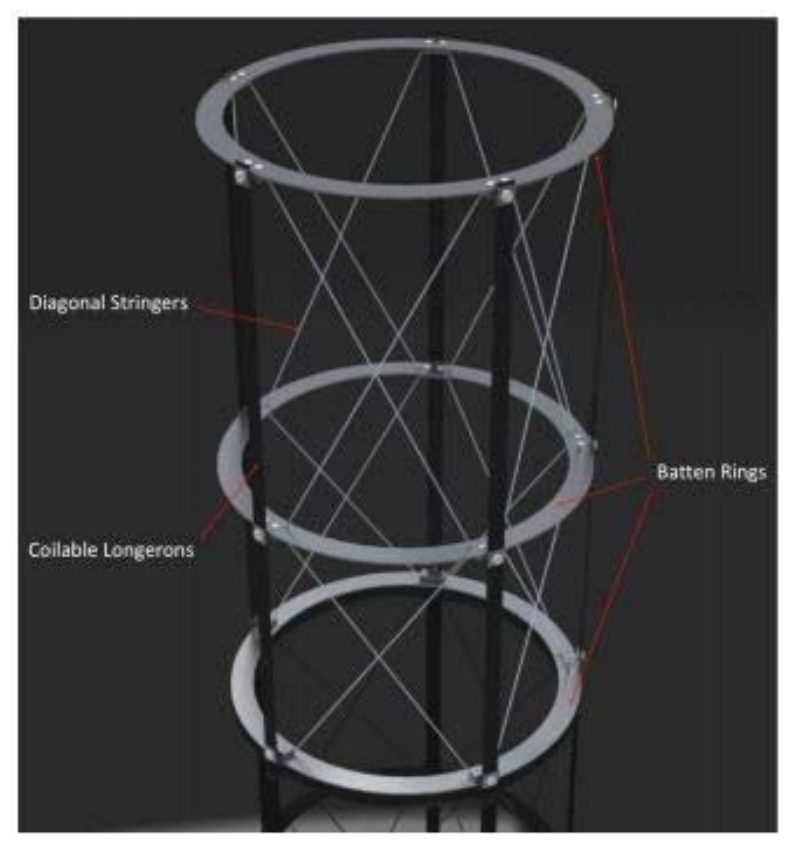

Figure 9: Concept Model of the Truss Skeleton

The Batten rings are structural members that transfer external loads to the longerons, and scatter light off of the telescope walls. The longerons, which are elastic and coil when stowed, are vital for both the deployment and support of the telescope. The telescope deploys passively due to the elastic properties of the longerons; when the constraints are released, these coiled longerons extend to their full length, shown in Figure 9. In addition, the longerons provide compressional strength to the truss. Finally, the diagonal stringers provide tensile strength, and have a low coefficient of friction for smooth deployment. Figure 10 shows the strain-deployed truss in the CubeSat, in both its stored form as well as its deployed form. 


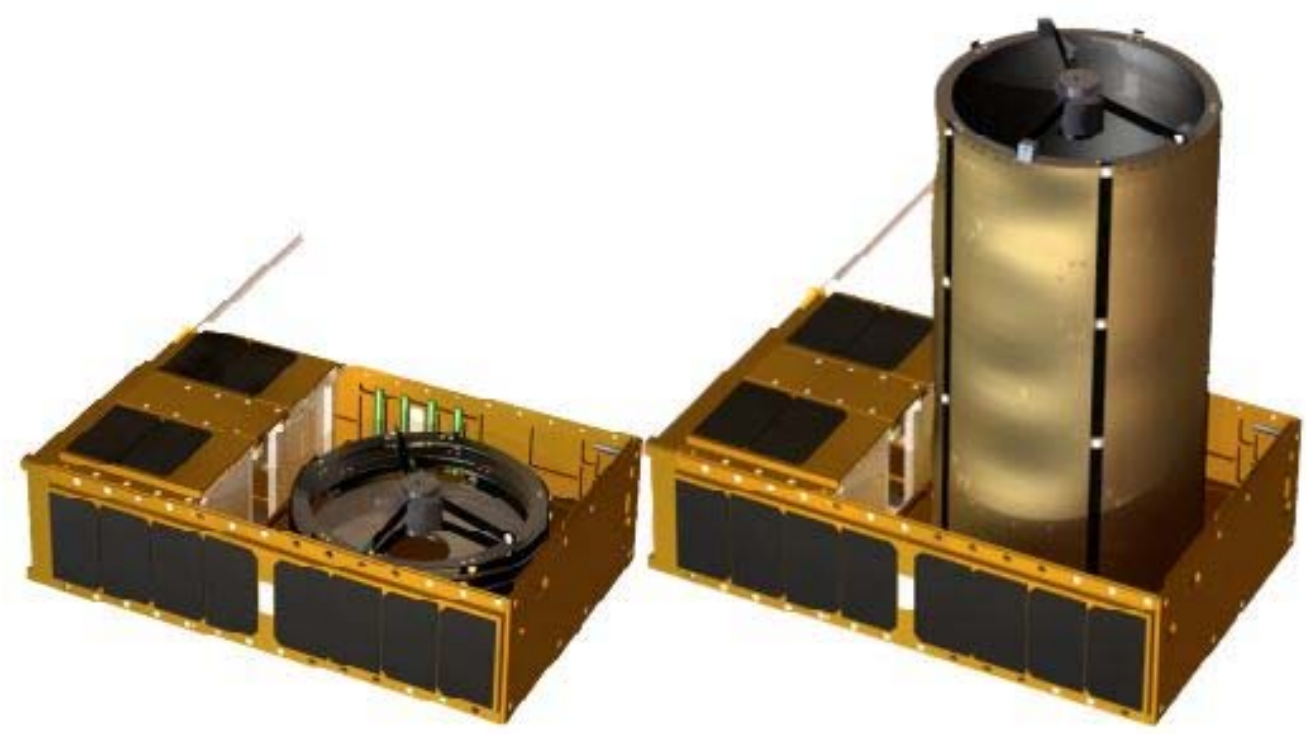

Figure 10. Drawing of Strain-Deployed Truss stowed (left) and deployed (right)

This configuration has the benefit of keeping the sieve flat and intact while stored, which is extremely important for the safety of the optics. In addition, the additional space allows for redundancy in the flight hardware, as well as room for components such as the laser. However, the size of the CubeSat itself is larger, which makes it more difficult to obtain an early launch manifest. Overall, this project focused on conceptual deployable technology for space imaging but the proposed components have not been constructed or tested, which increases the time needed prior to deployment. The concept is suited for the PS as it would allow the sieve to remain flat, eliminating material wrinkles that would be generated from flexible membrane.

\subsection{Photon sieve for Lidar application at NASA Langley Research Center}

NASA Langley Research Center (NASA LaRC) has been researching PS's for Lidar (Light Detection and Ranging) applications from 2015 [9,28-32]. Lidar is a similar measurement technique to Radar except using light pulses instead of radio waves, and uses reflected laser pulses to create images or collect science signal of distant particles and objects [33]. The light from the laser is a tightly coherent beam, which remains powerful compared with ordinary light even when traveling large distances. After the coherent beam reaches a region of interest, the reflected light can be filtered and analyzed to collect science 
signals or construct an image of the reflector objects. This kind of imagery is often used for small particles such as aerosols and $\mathrm{CO}_{2}$ (carbon dioxide) in the atmosphere, and has been used on NASA missions such as LITE (Lidar In-space Technology Experiment, 1994), CALIPSO (Cloud-Aerosol Lidar and Infrared Pathfinder Satellite Observation, 2006), CATS (Cloud-Aerosol Transport System, 2015) [34], and the upcoming GEDI (Global ecosystems dynamics investigation, 2018) [35]. NASA LaRC was the principal investigator for both LITE and CALIPSO, creating and testing the Lidar systems for Earth-observing applications.

From the benefit of higher signal-to-noise ratios, PS's are suitable for Lidar telescope lens application [3-7]. In addition, as the photon sieve focuses light of different wavelengths at different lengths automatically, there is no need to have an additional filter for the Lidar wavelength. The NASA LaRC PS project has two main goals. One goal is to improve science quality by eliminating sunlight contamination and backscatter noise in space-based Lidar measurements during daytime. Without a robust technique to discriminate the weak Lidar signals from strong sunlight contamination, the quantitative data from daytime Lidar measurements for optically thin compositions of the atmosphere have large uncertainties. The team at NASA LaRC developed a novel Lidar remote sensing concept that can eliminate sunlight-induced noise. For this goal, the NASA LaRC Lidar concept requires not only the photon sieve (PS) but also a vortex phase plate (VPP). When a transmitted laser light passes the VPP, the light carries orbital angular momentum (OAM) and moves to a photon sieve (PS) diffractive filter that separates scattered sunlight from laser light backscattered from the atmosphere, ocean and solid surfaces. Results of numerical modeling of the system show that after passing through the PS, laser light that carries the OAM is focused on a ring (called a "focal ring") on the focal plane of the PS filter. Due to the circularly polarized light nature, very little energy arrives at the center of the focal plane. However, scattered sunlight, as a plane wave without the OAM, focuses at the center of the focal plane and thus can be effectively blocked or ducted out as shown in Figure 11 [28]. Up to 6-inch diameter PS's have been manufactured in the advanced optical cleanroom at NASA LaRC [Figure 12] and used to experimentally validate the numerical model [28]. Another goal is the development of high diffraction efficiency PS's that can significantly reduce mission costs by replacing the traditional reflector telescope. The main drawback in using photon sieves is their inherently lower diffraction efficiency compared to zone plates. However, PS single and multilevel phase structures have recently been investigated and have reached efficiencies up to $25 \%$ [28-31]. 


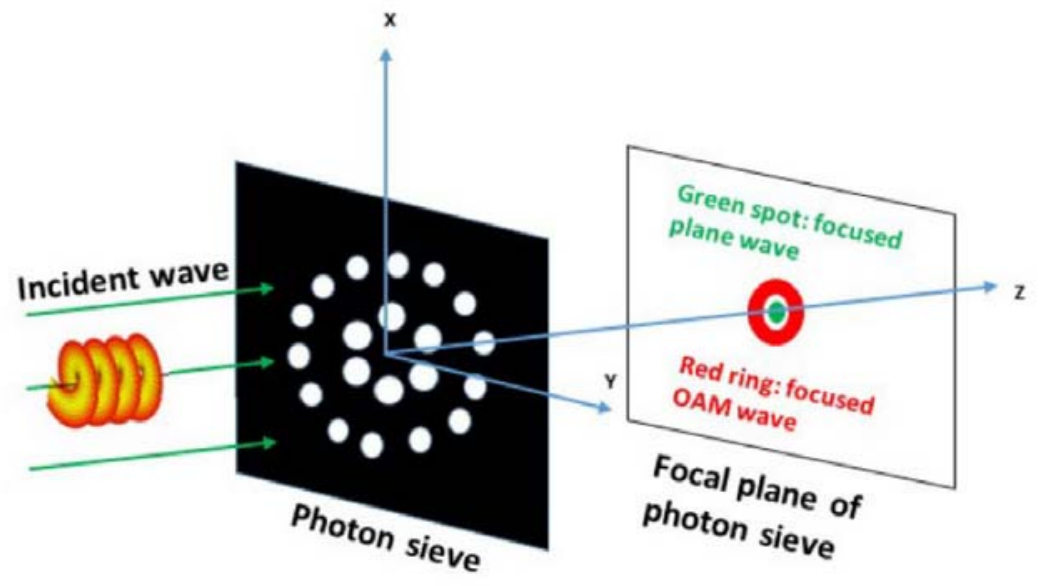

Figure 11. Illustration of a photon sieve and light of plane wave and light with the OAM focused on the focal plane [28].

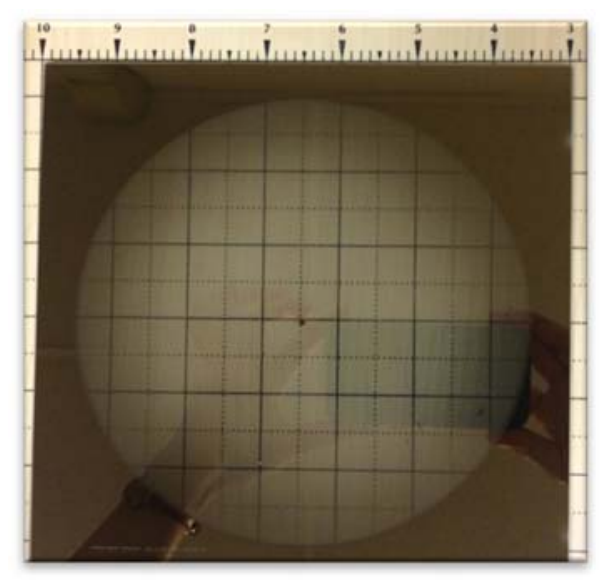

Figure 12. 6-inch diameter photon sieve on 7-inch chrome on soda lime glass substrate.

NASA LaRC introduced new applications of the PS and successfully demonstrated the possibility and advantage of the devices for Lidar telescope applications. However, due to laser source requirement for Lidar applications and CubeSat power limitations, a new CubeSat design for PS Lidar is necessary. Especially, the laser mounting on the photon sieve CubeSat itself is inefficient. More specifically, the laser sources for imaging or science signal collection require far too much power to be mounted on a CubeSat, as the solar panels only provide a small amount of power not used by the satellite's flight systems [36]. Finally, the high power requirement became necessary to plan for alternative laser sources or to modify the mission. Following are detail proposed ideas that would be able to place the photon sieve CubeSat within 
detection range of a laser source. On the proposed idea, the laser source is considered to be located within hundreds of meters to the CubeSat to keep the coherent nature and power of the return signal close to the laser source.

\section{(A) Satellite Constellation}

When multiple satellites are conducting measurements together, they are placed into a synchronous orbit called a satellite train or constellation traveling minutes or even seconds apart. These satellites are able to collaborate for a joint goal using a combination of their instruments. One such constellation, the Atrain, consists of six satellites that conduct Earth-observing atmospheric measurements [37]; one of the satellites, CALIPSO, is a $1064 \mathrm{~nm}$ Lidar satellite [38]. By entering a satellite constellation such as the Atrain, which has a Lidar instrument, the CubeSat would be close enough to the laser source to conduct measurements. There is a gap of more than 10 minutes between CALIPSO and Aura, the next satellite on the train, which another satellite could enter. However, one of the drawbacks of a CubeSat launch is that the location of orbit is entirely dependent on the location of the primary payload. Therefore, it is highly unlikely that this CubeSat will be able to enter a satellite constellation, as the destination of the primary payload will most likely differ. In addition, even if the primary payload is entering a satellite constellation, the CubeSat will be ejected in a manner that it is not a collision risk for the other satellites in orbit. Therefore, the CubeSat would be too far from the constellation to take advantage of the Lidar instruments. Adding the photon sieve to a Lidar satellite constellation is a possibility, but an unlikely one.

\section{(B) Using the Sun as a laser source}

In space, the Sun provides a massive amount of radiation in a spectrum of wavelengths. However, this white light differs from laser light and therefore does not provide the same signal capabilities. Laser light is coherent, which means that all of the light rays of the same wavelength travel in phase and in a tight manner [39]. This ensures that the beam remains focused and powerful over long distances, with little beam divergence. White light contains beams of many wavelengths traveling out of phase, which results in divergence and interference. Although it is possible to use a filter to isolate specific wavelengths of light, the beams would still be traveling out of phase, which would cause interference as well as divergence [40]. Using a filter followed by a focusing lens and then a collimating lens, which would in turn focus the beam and then make the rays parallel, the sunlight could be given the characteristics of a laser beam. These characteristics include parallel coherence rays, intense focus, and light of the same wavelength. The optical 
instruments used, such as the filter and the lens, are all compact enough to fit on a CubeSat, which makes this feasible in terms of size.

Although the sunlight could be focused in space itself to assume the characteristics of a laser, this technology would not be feasible for long distance imaging. By using lenses to focus and collimate the light, beam divergence is introduced, because of small defects in the lenses. Over small distances, these defects would not cause a noticeable change in the beam, but over the large distances needed for Earth observing, the beam would diverge greatly. This would cause weak return signals to use for photon sieves. Theoretically, perfect lenses would be able to focus and collimate the light with no divergence. Solar pumped laser work has been reported at NASA Langley Research Center [41-43]

\title{
(C) International Space Station Payload
}

The International Space Station (ISS) has a variety of exposed attachment sites for external payloads. One of the current mission payloads, the Cloud-Aerosol Transport System (CATS), utilizes a $1064 \mathrm{~nm}$ wavelength laser, which is the same laser used in the photon sieve project at NASA Langley. CATS will be deployed on the ISS until 2018 and will be replaced the same year by GEDI for LIDAR experiment. Both CATS and GEDI are/will be mounted on the Japanese Experiment Module - Exposed Facility (JEM-EF), which is one of the external payload attachment sites. As many launch vehicles carry cargo to the ISS, a photon sieve CubeSat could easily be sent to the station, where it could remotely be installed using the robotic arm on one of the payload attachments like JEM-EF. When the photon sieve telescope lens mounts at any location on the ISS, it would be close enough to read the signals from CATS and GEDI, as long as it was pointing in the correct direction.

The only problem with this solution is the limited amount of payload space on the ISS external attachments. However, this solution remains the most likely manner of placing a PS CubeSat within range of a laser light source.

\section{Potential Applications of PS and PS CubeSat}

\author{
a) EUV/IR Imaging
}


The versatility of photon sieves is one of the biggest advantages over conventional lenses. Because changing the design wavelength only requires a simple design change and choice of material, photon sieves can easily be adapted for use in any wavelength regime. Of particular interest for CubeSat applications are the EUV and IR ranges, which have applications in solar imaging and chemical remote sensing, respectively [44,45]. By enabling lightweight CubeSat imaging systems, photon sieves allow for low-cost, easy to manufacture alternatives to conventional optics in important astronomical and meteorological applications.

b) Near-field optics for spectroscopy, microscopy, and medical imaging

The optical near field is an area of great interest in recent years due to its wide variety of applications and sub-diffraction limit resolution [46]. However, refractive optics are not suitable candidates for near field optics due to the natural diffraction limit placed on a conventional curved lens. Therefore, planar and plasmonic optics are attractive alternatives for near field applications [47]. Recently, photon sieves have been demonstrated with plasmonic nanostructures for the purpose of independently focusing $s$ and $p$ polarizations at different focal planes [48]. By overlapping two photon sieves with different design wavelengths, each with a different "plasmonic scatterer" nanostructure, s- and ppolarizations were focused to different planes. In addition, metal/dielectric photon sieves could see an application as near field plasmonic devices in the event that the subwavelength sieve pinholes support extra-ordinary optical transmission (EOT) at the sieve design wavelength [49]. The feasibility of this effect is currently being studied by the authors. EOT in seen in periodic arrays of sub-wavelength holes in metal films on dielectric substrates, which is essentially what a photon sieve is if the design focal length is chosen to be sufficiently short. In the case that photon sieves support EOT, photon sieves would have a major advantage over conventional zone plates for optical near field applications, due to the signal enhancement from the EOT effect as well as the naturally superior image contrast of photon sieves.

c) Deep celestial and dark matter imaging with PS CubeSat 
Photon sieve space telescopes are also a large area of interest [3-7] due to their enhanced image contrast and low weight. However, further benefits exist as well. Photon sieves are more tolerant to folding and deformation prior to deployment, so integration of several meter diameter sieves onto a CubeSat platform is possible. In addition, with the advent of smart optical materials, mechanical compensation for membrane wrinkles or deformation is possible with using only a small voltage [50]. The use of bio-inspired designs is also possible in order to improve the folding and deployment of CubeSat photon sieves. The vein structure, the membrane, the material elasticity (resilin distribution) and the folding pattern play an important role for self-deployment and packaging in insect wings and are also inspiring for deployment concepts for large in-space membrane systems [51,52]. Based on the reference 48, the use of SWNTs as conductive additives in polymer matrices for space applications is advantageous for a number of reasons. Two major advantages in using these materials over conventional conductive fillers are their small size and high aspect ratio and transparency. Due to these features, relatively small amounts can be used to induce conductivity throughout the polymer matrix without significantly altering optical and mechanical properties. Furthermore, the predominantly membrane structures provide mission enabling geometric dimensions with very low mass/area (areal density) and they can be packed in small volumes for launch and transit. The cost savings of expandable structures have the potential to change the economics of space exploration and inexpensive CubeSat missions. However, these applications depend on the careful development and choice of appropriate sieve membrane materials. The temperature of the optical instrument (photon sieve) is influenced by the solar absorptance and thermal emittance of the materials. Optical and thermal properties can degrade over time in the harsh low earth orbital (LEO) space environment where the optical membrane materials are exposed to various forms of radiation, thermal cycling, and atomic oxygen. Therefore, it is important to test the durability of optical component materials in the space environment both Zenith and Nadir positions [53]. Especially, atomic oxygen (AO) is formed when $\mathrm{O}_{2}$ is broken apart by energetic UV radiation and the predominant species in LEO $(180 \sim 650 \mathrm{~km})$. Currently, Kapton polyimide is used for such applications [3-7].

d) Unique Industry Applications 
Photon sieves also have the potential to see unique industrial and military applications in areas such as nanolithography and enhanced weapons vision $[54,55]$. The photon sieves are used to focus UV laser light onto a photoresist, and lines resolution of $244 \mathrm{~nm}$, which can be further improved by increasing the NA of the photon sieve, or using a shorter lithography wavelength [54]. This application of photon sieves could become useful for instances when electron beam lithography would typically be needed, but at dimensions on the scale of $100 \mathrm{~nm}$, which would result in a lower processing cost. A team of researchers at the University of Florida proposed using photon sieves in a bio-inspired insect eye format in order to improve weapon systems vision [55]. By using multiple photon sieves in an insect-eye layout, the field of view is greatly enhanced, allowing for imaging of the target and surroundings as opposed to just the target alone.

\section{Conclusion}

The photon sieve is a revolutionary, lightweight diffractive optic that can change the standard of space imaging and science signal detection. As it is a flexible membrane, it can easily be mounted on a CubeSat, following either the $3 \mathrm{U}$ or the $6 \mathrm{U}$ size standards. There have been multiple projects dealing with the use of deployable photon sieves (PS) for CubeSat applications. Although there is no current flight heritage with photon sieves, the FalconSAT-7, scheduled to launch in 2018, will confirm the feasibility of the photon sieve technology. However, the other technology concepts require further testing in order to be considered flight-ready. This includes microgravity testing (already completed by the FalconSAT-7), finite element analysis, vibration/shock testing, and thermal-vacuum testing. The electronic and optical components of the CubeSat also need to undergo radiation testing and physical properties test in vacuum similar environment of space to ready for space flight. Using Lidar technology, the photon sieve will be able to conduct Earth imaging or collect science signal at a fraction of the cost of traditional telescope missions. While the laser used cannot be mounted on the CubeSat itself, there are

alternatives such as flying in a satellite constellation or mounting the CubeSat as a payload on the ISS. Using these new capabilities as well as previous success with Sun-imaging PS CubeSats, there is the possibility to produce a multi-faceted photon sieve-imaging mission. PS also can use for 
EUV/IR imaging, near-field optics for spectroscopy, microscopy, and medical imaging, and enhanced weapon vision systems for industry and military.

\section{Acknowledgement}

This research was supported by photon sieve Internal Research And Development (IRAD) program at NASA Langley Research Center. The authors appreciate Dr. Wenbo Sun, Dr. Yongxiang Hu, Dr. Ken Tedjojuwono, and Dr. William Humphreys for their help, fruitful discussion, and support during the work.

\section{References}

[1] L. Kipp, M. Skibowski, R. L. Johnson, R. Berndt, R. Adelung, S. Harm, and R. Seemann, "Sharper images by focusing soft-X-rays with photon sieves", Nature, 414, 184-188, 2001

[2] G.Andersen, "Large optical photon sieve," Opt. Lett. 30, 2976-2978 (2005).

[3] G. Andersen, O. Asmolova, G. McHarg, M. Dearborn and T. Quiller, "FalconSAT-7: Towards rapidly deployable space-based surveillance," AMOS (Advanced Maui Optical and Space Surveillance) Technologies Conference, Wailea Beach, 2013.

[4] O. Asmolova, G. Andersen, M. E. Dearborn, M. G. McHarg, T. Quiller and T. Murphey, "Optical analysis of a membrane photon sieve space telescope," United States Air Force Academy, 2013.

[5] G. Andersen, O. Asmolova, M. G. McHarg, T. Quiller and C. Maldonado, "FalconSAT-7: A membrane space solar telescope," SPIE 9143, Space Telescopes and Instrumentation 2014: Optical, Infrared, and Millimeter Wave, Montréal, Quebec, Canada, 2014. 
[6] M. E. Dearborn, G. P. Andersen, O. Asmolova, R. L. Balthazor, M. G. McHarg, H. C. Nelson, T. S. Quiller, G. R. Wilson, T. J. Harvey and T. W. Murphey, "A Deployable Membrane Telescope Payload for CubeSats," Journal of Small Satellites, 2014.

[7] B. Smith, "FalconSAT-7 Deployable Solar Telescope," United States Air Force Academy, Colorado Springs, Colorado, 2014.

[8] Geoff Andersen, "Membrane photon sieve telescopes," Appl. Opt. 49, 6391-6394 (2010).

[9] M. Julian, D. Macdonnell, and M. Gupta, "High efficiency multilevel photon sieves", in preparation (2018).

[10] California Polytechnic State University, "CubeSat Design Specification, Rev.13," California Polytechnic State University, San Luis Obispo, 2014.

[11] California Polytechnic State University, "6U CubeSat Design Specification Rev. PROVISIONAL," California Polytechnic State University, San Luis Obispo, CA, 2016.

[12] "LAICE (Lower Atmosphere/Ionosphere Coupling Experiment)," [Online]. Available: https://directory.eoportal.org/web/eoportal/satellite-missions/l/laice.

[13] G. D. Krebs, "RBLE (Radiation Belt Loss Experiment)," [Online]. Available: http://space.skyrocket.de/doc sdat/rble.htm.

[14] S. Spremo, E. Taylor and D. Tomko, "E. coli AntiMicrobial Satellite," NASA Ames Research Center, Moffett Field, CA, 2015.

[15] G. D. Krebs, "Rascal (SLU 04)," [Online]. Available: http://space.skyrocket.de/doc sdat/rascal.htm.

[16] Boston University, "Andesite," [Online]. Available: http://www.bu.edu/busat/missions/andesite/.

[17] S. C. Reising, T. C. Gaier, C. D. Kummerow, V. Chandrasekar, S. T. Brown, S. Padmanabhan, B. H. Lim, S. C. van den Heever, T. S. L'Ecuyer, C. S. Ruf, Z. S. Haddad, Z. J. Luo, S. J. Munchak, T. C. Koch 
and S. A. Boukabara, "Temporal Experiment for Storms and," NASA Earth Science Technology Forum, Pasadena, CA, 2015.

[18] E. Peral, S. Tanelli, Z. Haddad, O. Sy, G. Stephens and E. Im, "RainCube: A Proposed Constellation of Precipitation Profiling Radars," IEEE Geoscience and Remote Sensing Society, Milan, Italy, 2015.

[19] M. Pilinksi, E. Stromberg, C. Fish, G. Crowley, C. Huang, P. Roddy, L. Gentile, R. Heelis, R. Stoneback, A. Vera, C. Kief, B. Zufelt and J. Retterer, "Scintillation Observations and Response of," 13th Annual CubeSat Developer's Workshop, San Luis Obispo, CA, US, 2016.

[20] J. T. Johnson, C.-C. Chen, C. Ball, A. O'Brien, L. Garry, M. Andrews, C. McKelvey, G. Smith, S. Misra, S. Brown, J. Kocz, B. Jarnot, J. Piepmeir, J. Lucey, P. Mohammed, D. Bradley, K. Horgan and M. Solly, "CubeRRT: CubeSat Radiometer RFI Technology," NASA Earth Science Technology Forum, Annapolis, Maryland, 2016.

\section{[21] https://www.nasa.gov/mission_pages/smallsats}

[22] W. K. Belvin, M. E. Zander, D. W. Sleigt, J. Connell, N. Holloway, and F. Palmieri, "Materials, structures and manufacturing: An integrated approach to develop expandable structures", AIAA

[23] W. K. Belvin, M. Straubel, W. K. Wilkie, M. E. zander, J. M. Fernandez, and M. F. Hillebrandt, "Advanced deployable structural systems for small satellites"

[24] E. Agasid, R. Burton, R. Carlino, G. Defouw, A. Dono Perez, A. G. Karacalığlu, B. Klamm, A. Rademacher, J. Schalkwyck, R. Shimmin, J. Tilles and S. Weston, "Small Spacecraft Technology State of the Art," NASA, Moffett Field, CA, 2015.

[25] CubeSat 101: Basic concepts and processes for first-time CubeSat developers, NASA, 2018

[26] T. W. Murphey and J. N. Footdale, "Design and Testing of Self-Deploying Membrane Optic Support Structure Using Rollable Composite Tape Springs," Proceedings of the 54th AIAA Structures, Structural Dynamics, and Materials Conference, Boston, MA, 2013.

[27] E. Agasid, A. Rademacher, M. McCullar and R. Gilstrap, "Study to Determine the Feasibility of a Earth Observing Telescope Payload for a 6U Nano Satellite," NASA Ames Research Center, Moffett Field, California, 2010. 
[28] W. Sun, Y. Hu, D. G. Macdonnell, C. Weimer, and R. R. Baize, "Technique to separate lidar signal and sunlight”, Optical Express 12949, 2016

[29] Wenbo Sun, Yongxiang Hu, David G. MacDonnell, Hyun Jung Kim, Carl Weimer, and Rosemary R. Baize, "Fully reflective photon sieve" Journal of Quantitative Spectroscopy \& Radiative Transfer 206 , 101-104, February 2018

[30] Wenbo Sun, Yongxiang Hu, David G. MacDonnell, Carl Weimer, Hyun Jung Kim, and Rosemary R. Baize, "A fully transparent photon sieve" Optics Express 25 (15), 17356-17363, 2017

[31] M. N. Julian, D. G. Macdonnell, and M. C. Gupta, "Fabrication of photon sieves by laser ablation and optical properties", Optics Express, 25, 31528, 2017.

[32] Shravan Hariharan, Sean Fitzpatrick, Matthew Julian, Hyun Jung Kim, and David Macdonnell, "Rapid generation of large dimension phonon sieve designs", NASA/TM, NASA/TM-2017

[33] M. P. McCormick, "LITE: Measuring the Atmosphere with Laser Precision," NASA, 1 August 1994. [Online]. Available: https://www.nasa.gov/centers/langley/news/factsheets/LITE.html. [Accessed 6 July 2017].

[34] J. E. Yorks, M. J. McGill, D. L. Hlavka, W. D. Hart, S. P. Palm, E. Nowottnick, M. A. Vaughan, P. Selmer, S. Rodier, P. R. Colarco, A. da Silva and V. Buchard-Marchant, "New Capabilities for Space-Based Cloud and Aerosols Measurements: The Cloud-Aerosol Transport System (CATS)," NASA, 2014

[35] R. Dubayah, "GEDI LIdar: Global Ecosystem Dynamics Investigation," POLinSAR 2015 and 1st BIOMASS Workshop, ESA/ESRIN, Frascati, Italy, 2015.

[36] D. Selva and D. Krejci, "A survey and assessment of the capabilities of Cubesats for Earth observation," Acta Astronautica, 2012.

[37] NASA Goddard Space Flight Center, "Formation Flying: The Afternoon "A-Train" Satellite Constellation," NASA, Greenbelt, Maryland, 2003.

[38] D. Winker, J. Pelon, J. Coakley, S. Ackerman, R. Charlson, P. Colargo, P. Flamant, Q. Fu, R. Hoff, C. Kittaka, T. Kubar, H. Le Treut, M. McCormick, G. Mégie, L. Poole, K. Powell, C. Trepte, M. Vaughan and B. Wielicki, "The CALIPSO Mission: A Global 3D View of Aerosols and Clouds," American Meteorogical Society, 2010.

[39] NASA Langley Research Center, "Laser Technology: Shedding Some Light," NASA, 10 December 
1996. [Online]. Available: https:/www.nasa.gov/centers/langley/news/factsheets/LaserTech.html. [Accessed 10 July 2017].

[40] P. D. Reusswig, S. Nechayev, J. M. Scherer, G. W. Hwang, M. G. Bawendi, M. A. Baldo, and C. Rotschild, "A path to practical solar pumped lasers via radiative energy transfer", Scientific Report, DOI: 10.1038/srep14758, 2015.

[41] Ja H. Lee and W. R. Weaver, "A solar simulator-pumped atomic iodine laser”, Appl. Phys. Lett. 39, 137 (1998).

[42] S. Mizuno, H. Ito, K. Hasegawa, T. Suzuki, and Y. Ohishi, "Laser emission from a solar-pumped fiber”, Optics Express, 20 (6), 5891 (2012).

[43] T. Mzsuda, M. Iyoda, Y. Yasumatsu, and M. Endo, "Low-concentrated solar-pumped laser via transverse excitation fiber-laser geometry”, Optics Letters, 41 (17), 3427 (2017).

[44] J.-P. Delaboudiniere, R.A. Stern, A. Maucherat, F. Portier-Fozzani, W.M. Neupert, J.B. Gurman, R.C. Catura, J.R. Lemen, L. Shing, G.E. Artzner, J. Brunaud, A.H. Gabriel, D.J. Michels, J.D. Moses, B. Au, K.P. Dere, R.A. Howard, R. Kreplin, J.M. Defise, C. Jamar, P. Rochus, J.P. Chauvineau, J.P. Marioge, F. Clette, P. Cugnon, E.L. Van Dessel, "Imaging the solar corona in the EUV," Advances in Space Research, Volume 20, Issue 12, (1997).

[45] Al-Saadi, Jassim; Szykman, James; Pierce, R. Bradley; Kittaka, Chieko; Neil, Doreen; Chu, D. Allen; Remer, Lorraine; Gumley, Liam; Prins, Elaine; Weinstock, Lewis; MacDonald, Clinton; Wayland, Richard; Dimmick, Fred; Fishman, Jack, "Improving National Air Quality Forecasts with Satellite Aerosol Observations," Bulletin of the American Meteorological Society, vol. 86, Issue 9, pp.1249-1261 (2005).[46] D Courjon and C Bainier, "Near field microscopy and near field optics," Reports on Progress in Physics, 57 (10), (1994).

[47] Ekmel Ozbay, "Plasmonics: Merging Photonics and Electronics at Nanoscale Dimensions," Science Vol. 331, Issue 5758, pp. 189-193, (2006).

[48] C. Williams, Y. Montelongo, T.D. Wilkinson, Plasmonic metalens for dual-focus narrowband imaging, Advanced Optical Materials, 5 (24) (2017).

[49] T. W. Ebbesen; H. J. Lezec; H. F. Ghaemi; T. Thio; P. A. Wolff, "Extraordinary optical transmission through sub-wavelength hole arrays," Nature, 391, 667-669 (1998).

[50] S. Lee, A. Reuveny, J. Reeder, S. Lee, Ha. Jin, Q. Liu, T. Yokota, T. Skitani, T. Isoyama, Y. Abe, Z. Suo, and T. Someya, "A transparent bending-insensitive pressure sensor”, Nature nanotechnology, 11, 472 (2016). 
[51] F. Haas, S. Gorb, and R. J. Wootton, "Elastic joints in dermapteran hind wings: materials and wing folding, In: Arthropod Structure \& Development, Vol. 29, p, 137-146, Elsevier Science Ltd., 2000

[52] D. M. Delozier, J. W. Connell, J. G. Smith, and K. A. Watson, "Preparation and characterization of space durable polymer nanocomposite films from functionalized carbon nanotubes

[53] Y. Li, K. K. De Groh, B. A. Banks, H. Leneghan, and O. Asmar, "Effect of Ram and Zenith exposure on the optical properties of polymers in space"

[54] Rajesh Menon, Dario Gil, George Barbastathis, and Henry I. Smith, "Photon-sieve lithography," J. Opt. Soc. Am. A 22, 342-345 (2005).

[55] K. Kincade, Laser Focus World 40, 34 (2004). 